Provided for non-commercial research and education use. Not for reproduction, distribution or commercial use.

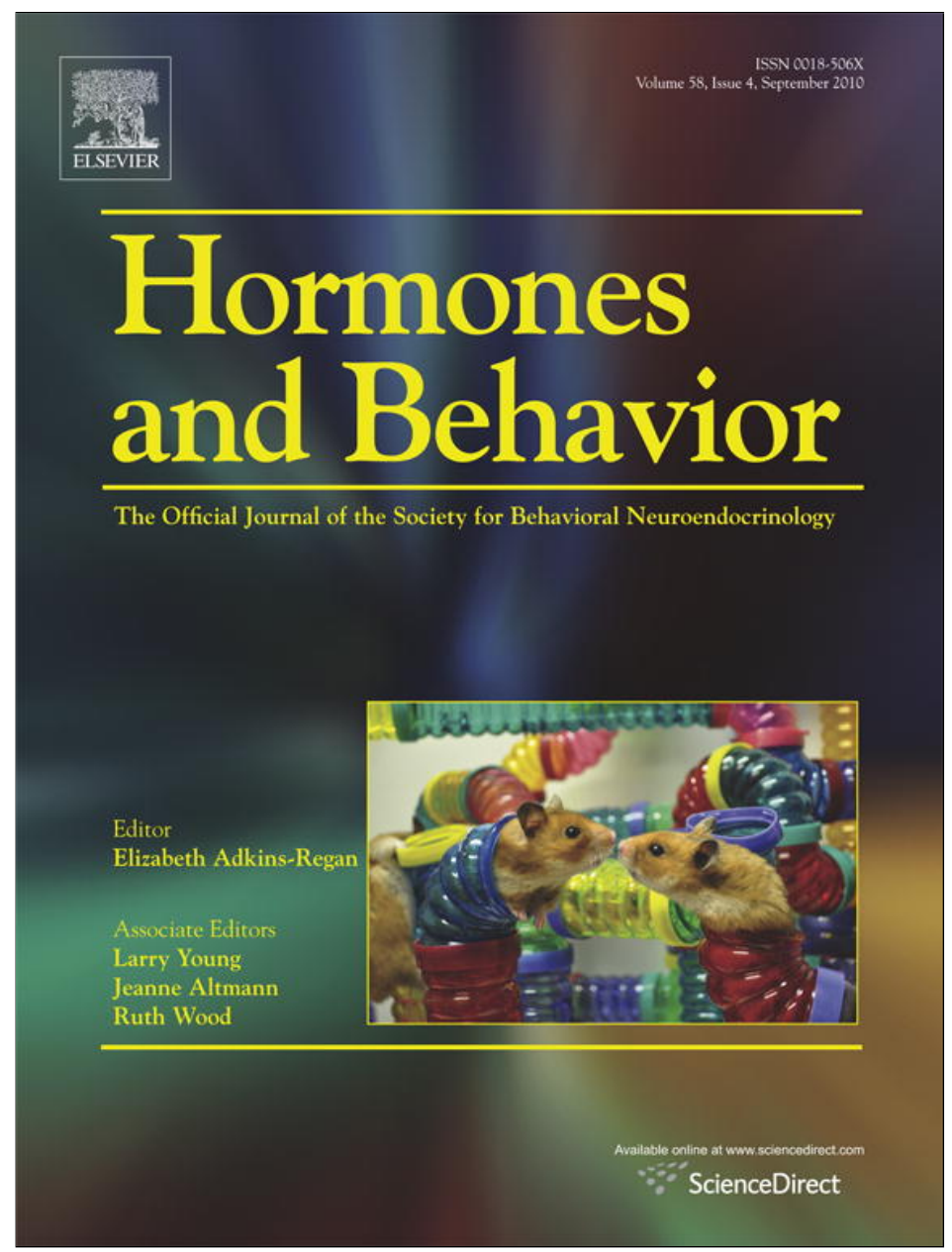

This article appeared in a journal published by Elsevier. The attached copy is furnished to the author for internal non-commercial research and education use, including for instruction at the authors institution and sharing with colleagues.

Other uses, including reproduction and distribution, or selling or licensing copies, or posting to personal, institutional or third party websites are prohibited.

In most cases authors are permitted to post their version of the article (e.g. in Word or Tex form) to their personal website or institutional repository. Authors requiring further information regarding Elsevier's archiving and manuscript policies are encouraged to visit:

http://www.elsevier.com/copyright 


\title{
Sudden weather deterioration but not brood size affects baseline corticosterone levels in nestling Alpine swifts
}

\author{
Pierre Bize ${ }^{\mathrm{a}, *}$, Antoine Stocker $^{\mathrm{a}}$, Susanne Jenni-Eiermann ${ }^{\mathrm{b}}$, Julien Gasparini ${ }^{\mathrm{c}}$, Alexandre Roulin ${ }^{\mathrm{a}}$ \\ a Department of Ecology \& Evolution, University of Lausanne, Biophore, CH-1015 Lausanne, Switzerland \\ b Swiss Ornithological Institute, CH-6204 Sempach, Switzerland \\ c Laboratoire de Parasitologie Evolutive, Université Pierre et Marie Curie, CNRS UMR 7103,7 quai St-Bernard, 75252 Paris, France
}

\section{A R T I C L E I N F O}

\section{Article history:}

Received 28 June 2010

Revised 28 June 2010

Accepted 30 June 2010

Available online 8 July 2010

\section{Keywords:}

Baseline corticosterone

Brood size manipulation

Sibling competition

Unpredictable perturbations

Weather conditions

\begin{abstract}
A B S T R A C T
While evidence is accumulating that stress-induced glucocorticoid responses help organisms to quickly adjust their physiology and behaviour to life-threatening environmental perturbations, the function and the ecological factors inducing variation in baseline glucocorticoid levels remain poorly understood. In this study we investigated the effects of brood size by experimentally manipulating the number of nestlings per brood and the effect of weather condition on baseline corticosterone levels of nestling Alpine swifts (Apus melba). We also examined the potential negative consequences of an elevation of baseline corticosterone on nestling immunity by correlating corticosterone levels with ectoparasite intensity and the antibody production towards a vaccine. Although nestlings reared in enlarged broods were in poorer condition than nestlings reared in reduced broods, they showed similar baseline corticosterone levels. In contrast, nestling baseline corticosterone levels were higher immediately after cold and rainy episodes with strong winds. Neither nestling infestation rate by ectoparastic flies nor nestling antibody production against a vaccine was correlated with baseline corticosterone levels. Thus, our results suggest that altricial Alpine swift nestlings can quickly modulate baseline corticosterone levels in response to unpredictable variations in meteorological perturbation but not to brood size which may be associated with the degree of sibling competition. Apparently, short-term elevations of baseline corticosterone have no negative effects on nestling immunocompetence.
\end{abstract}

(c) 2010 Elsevier Inc. All rights reserved.

\section{Introduction}

Endocrine mechanisms are central in regulating the allocation of resources among life history traits such as self-maintenance, growth and reproduction (Ricklefs and Wikelski, 2002; Zera and Harshman, 2001). Evidence is accumulating that glucocorticoid hormones (i.e. primarily cortisol in fishes and most mammals, and corticosterone in amphibians, reptiles and birds) released by the hypothalamopituitary-adrenal (HPA) axis are important modulators of vertebrate life histories (Bonier et al. 2009; Landys et al., 2006; McEwen and Wingfield, 2003; Romero, 2004; Wada, 2008; Wingfield and Kitaysky, 2002). Circulating levels of glucocorticoids are commonly divided in two categories. Baseline levels which are characterised by low glucocorticoid concentrations and slow within-individual variation over time (i.e. daily to seasonal rhythms; Romero, 2002), and stressinduced levels characterised by high glucocorticoid concentrations released within a few minutes after exposure to a stressful stimulus such as the presence of a predator or a standardised capture and

\footnotetext{
* Corresponding author. Fax: +41216924165.

E-mail address: pierre.bize@unil.ch (P. Bize).
}

handling procedure by the experimenter (i.e. the "stress response"; Müller et al., 2009; reviewed in Landys et al., 2006; Romero, 2004; Wingfield and Kitaysky, 2002). The stress response has been demonstrated repeatedly to induce a large variety of behavioural and physiological effects, such as high foraging behaviour, escape behaviour, and increased gluconeogenesis and protein catabolism (reviewed in Sapolsky et al., 2000; Wingfield and Kitaysky, 2002; Romero, 2004; Breuner et al. 2008). The stress response is viewed as being adaptive, allowing individuals to quickly respond to unpredictable stressful stimuli by resetting their physiology and behaviour to the new prevailing environmental conditions and thereby to reallocate resources to life history traits which support immediate survival (e.g. Blas et al., 2007; Romero and Wikelski, 2001). Yet, long lasting exposure to elevated amount of glucocorticoids can have long term fitness costs since it diverts resources from life history traits that are essential for long-term survival (reviewed in Sapolsky et al., 2000; Wingfield and Kitaysky, 2002; Romero, 2004). A well known negative effect of exposure to elevated amount of glucocorticoids is immunosuppression, and as a consequence greater susceptibility to diseases (Yang and Glaser 2002). In contrast to the stress response, ecological factors inducing variation in baseline glucocorticoid levels have received far less attention (but see Love et al., 2003; Blas et al., 
2005). The costs and benefits associated with variation in baseline glucocorticoid levels are less well understood (Landys et al., 2006; Romero, 2002). Indeed, variation in baseline glucocorticoid levels have been reported to be positively, negatively, or non-significantly related to estimates of fitness (Bonier et al. 2009).

The aims of the present study were to examine the effects of brood size and weather condition on variation in baseline corticosterone levels of wild nestling Alpine swifts (Apus melba). We also investigated the potential negative consequences of an elevation of baseline corticosterone levels on nestling immune function. Altricial offspring rely entirely on their parents for food, of which parents can only bring limited amount to their brood. As a consequence, offspring may have to beg and jostle vigorously for food, with competition levels increasing with the number of siblings per brood (Leonard et al., 2000). Experiments where offspring were given corticosterone implants demonstrated that elevated levels of exogenous baseline corticosterone trigger higher begging rates in the black-legged kittiwake (Rissa tridactyla; Kitaysky et al., 2001b), and cross-fostering experiments showed that parental provisioning rate is related to endogenous baseline corticosterone levels and spontaneous begging intensity in nestling thin-billed prions (Pachyptila belcheri; Quillfeldt et al., 2006). Thus, one hypothesis is that elevation of baseline corticosterone helps individuals to deal with nutritional deficits that result from sibling competition. This hypothesis predicts that offspring in large broods will have higher levels of circulating baseline corticosterone than offspring in small broods, assuming that elevated baseline corticosterone induces begging in nestlings raised in large broods. In agreement with this hypothesis, a brood size manipulation experiment performed in the barn swallow (Hirundo rustica) showed that nestlings reared in enlarged broods, and therefore facing increased social competition, had higher baseline corticosterone levels than nestlings in reduced broods (Saino et al., 2003). Yet, two recent experimental studies reported no difference in baseline corticosterone levels between brood size treatments in nestling spotless starlings (Sturnus unicolor; Gil et al., 2008) and nestling blue tits (Cyanistes caeruleus; Lobato et al., 2008). Thus, whether an increase of sibling competition is coupled with an increase in baseline corticosterone levels is currently unclear and further study is needed.

Climatic conditions have strong effects on food availability and, as a consequence, on development, reproductive success and survival. Evidence is accumulating that nutritional stress triggers the upregulation of the HPA axis in growing or adult organisms (JenniEiermann et al., 2008; Kitaysky et al., 1999b; Kitaysky et al., 2001a; Lobato et al., 2008; Pravosudov et al., 2001; Pravosudov and Kitaysky, 2006). These high corticosterone levels have been proposed to help individuals to mobilise body reserves and induce foraging behaviour (Landys et al., 2006). Thus, a second non-mutually exclusive hypothesis is that an elevation of baseline corticosterone helps individuals to survive prolonged periods of nutritional deficit caused by inclement climatic conditions. This hypothesis predicts that individuals will have higher levels of baseline corticosterone during periods of inclement weather, as recently observed in adult barn swallows (Jenni-Eiermann et al., 2008), nestling blue tits (C. caeruleus) and nestling pied flycatchers (Ficedula hypoleuca) (Lobato et al., 2008).

The Alpine swift is a suitable study system to investigate the effects of brood size and weather condition on nestling variation in baseline corticosterone levels for the following reasons. Firstly, the Alpine swift produces one brood of 1 to 4 altricial nestlings per year that compete for food resources until they are fully fledged, which occurs 50 to 70 days after hatching (Bize et al., 2004). Thus, we investigated whether baseline corticosterone levels were elevated in response to sibling competition by creating experimentally reduced and enlarged broods. Swifts usually reproduce in cavities and nestlings jostle for access to food provisioned by parents by approaching the cavity entrance (Malacarne et al. 1994; Bize and Roulin, 2006a). It has been demonstrated elsewhere that nestling Alpine swifts reared in enlarged broods are more likely to fall from the nest (Bize and Roulin, 2006a), which indicates that brood size manipulation is effective at increasing competition among Alpine swift siblings. We predicted that, if an elevation of baseline corticosterone levels favours the acquisition of food from parents under increased sibling competition, nestlings reared in enlarged broods would have higher baseline corticosterone levels than nestlings in reduced broods. Secondly, the Alpine swift feeds exclusively on aerial insects and offspring are forced to fast for several consecutive hours to days during unpredictable periods of inclement weather (Arn, 1960; Bize et al., 2006). We evaluated whether nestling Alpine swifts adjust their baseline corticosterone levels in response to inclement climatic conditions (as measured by cold temperatures, spells of rain and wind bursts) which negatively affect flying insect abundance (Grüebler et al., 2008) and in turn adult foraging performance (Cucco et al., 1992). We predicted that, if an elevation of baseline corticosterone helps nestlings to survive a weather-induced period of nutritional deficit, nestlings should up-regulate their baseline corticosterone levels when facing harsh weather. Finally, because Alpine swift nestlings are heavily infested by blood sucking louse flies (Crataerina melbae, Hippoboscidae, Diptera) that impair their development (Bize et al., 2003) and survival (Bize et al., 2005), we also investigated whether an elevation of baseline corticosterone levels comes at a cost of nestling humoral immunity. At the same ages (i.e. days 20 and 40 after hatching) when we measured baseline corticosterone levels, we also measured nestling ectoparasite load and antibody production against a vaccine. We predicted that, if an elevation of baseline corticosterone depresses the immune system, nestlings showing elevated baseline corticosterone levels should mount a weaker humoral immune response and they should be infested by greater numbers of louseflies. Because circulating corticosterone levels and the ability to respond to a stressor can increase with age in developing birds (i.e. 'the developmental hypothesis'; Sims and Holberton, 2000), we investigated the relationships between baseline corticosterone levels and brood size manipulation, weather conditions and the immune system both early and late in the development of nestling Alpine swifts (i.e. days 20 and 40 after hatching). We predicted that, if the capacity of nestlings to respond to stress developed gradually with post-hatching development, the relationships between baseline corticosterone levels and brood size manipulation, weather conditions and the immune system should be stronger late than early in the development.

\section{Materials and methods}

\section{Brood size manipulation experiment}

Fieldwork was performed in 2006 in an Alpine swift colony of ca. 100 breeding pairs located under the roof of a clock tower in Bienne, Switzerland. Throughout the breeding season (May-August), nests were visited daily to record laying date, clutch size and hatching date of the first egg denoted 'day 0'. We matched 15 pairs of nests containing two to three nestlings that hatched at the same date. Experimental nestlings hatched between the 16th June and the 7th July 2006, which covered the entire hatching period in this colony. Two days after hatching we exchanged two nestlings against one between matched nests. Thus, brood size was reduced by one nestling in half of the nests, while in the other half of the nests brood size was enlarged by one nestling. Nestlings were individually recognised by marking them with non-toxic coloured markers at the time of crossfostering before ringing them with a numbered aluminium ring at 10 days of age. In total, we exchanged 45 nestlings while 27 other nestlings remained in their natal nest. Prior to manipulation each nest contained two to three nestlings, and thus after the manipulation brood size remained within the natural range with broods containing 
a maximum of four nestlings (i.e. 8 one-nestling broods and 7 twonesting broods in the reduced treatment, and 10 three-nestling broods and 5 four-nestling broods in the enlarged treatment). At the time of cross-fostering, exchanged nestlings were similar in mass and wing length to their resident siblings (paired $t$-test, all $P>0.40$ ) and as their new nest-mates (paired $t$-test, all $P>0.26$ ). At the time of crossfostering enlarged and reduced broods did not differ in clutch size, hatching date, brood size and mean nestling body mass (all $P$ values $>0.58$ ). At fledging, there were more nestlings in enlarged than reduced broods (mean \pm SE brood size in enlarged vs. reduced broods $=3.33 \pm 0.13$ vs. $1.47 \pm 0.13$; Wilcoxon test, $z=4.82$, $P<0.0001)$ despite a significantly higher mortality of nestlings in enlarged than reduced broods (mean \pm SE proportion of nestling mortality in enlarged vs. reduced broods $=31.7 \pm 7.1 \%$ vs. $10.0 \pm 7.2 \%$; General linear model with a binomial distribution and a logit link, $\left.\chi^{2}=4.88, P=0.027\right)$. Nestlings died at similar ages in enlarged vs. reduced broods ( $35.2 \pm 5.1$ days vs. $36.2 \pm 15.2$; $t$-test assuming unequal variance, $t=-0.06, P=0.96)$.

\section{Measurements of nestlings, vaccination and blood sampling}

At day 15, all nestlings involved in the study were injected subcutaneously in the neck with $0.1 \mathrm{ml}$ of the TETRAVAC@ vaccine (Aventis Pasteur MSD, Switzerland; diphtheria 30 UI, tetanus 40 UI, pertussis $25 \mu \mathrm{g}$, filamentous haemagglutinin $25 \mu \mathrm{g}$, type 1 poliovirus $\mathrm{D}$ antigen 40 units, type 2 poliovirus D antigen 8 units and type 3 poliovirus D antigen 32 units). The TETRAVAC@ vaccine induces the production of antibodies against four antigens, which allows us to test whether nestling primary humoral immune responses towards different antigens were compromised by brood size and poor weather conditions (Gasparini et al., 2009). Before injection, we collected a blood sample $(60-120 \mu \mathrm{l})$ to measure pre-existing TETRAVAC-antigens. At days 20 and 40, we collected blood samples for measurements of plasma corticosterone and anti-TETRAVAC antibodies using the following standardised protocol. Nestlings were removed from the nest and a blood sample was collected within a time range of 24$360 \mathrm{~s}$ ( mean $\pm \mathrm{SE}=105 \pm 65 \mathrm{~s}$ ) after initial disturbance. Because the Alpine swift is a colonial bird, we took care to avoid disturbing nestlings before blood sampling. Nests are located in different rooms of the clock tower in Bienne, and thus focal nestlings were always blood sampled the first time of the day we entered the room. The blood was taken from the alar vein in a system for capillary blood collection (SARSTEDT, Microvette ${ }^{\circledR}$ CB 300, Lithium heparin). Blood samples were centrifuged in the field within $10 \mathrm{~min}$ after blood collection and plasma and red cells were immediately stored at $-20{ }^{\circ} \mathrm{C}$ before analyses. Nestlings were weighed to the nearest $0.1 \mathrm{~g}$, and the length of the left wing was measured to the nearest $\mathrm{mm}$ and sternum length to the nearest $0.1 \mathrm{~mm}$. We also counted the number of louse-flies located in the plumage of each nestling before putting nestlings back to their nest. Sampling occurred between 11:00 and 18:00 (median $=13: 00$ ). We determined the sex of each nestling by extracting nuclear DNA from the red cells and by amplifying sexspecific markers with a PCR method (Bize et al. 2005). The brood size manipulation experiment, vaccination and blood sampling were carried out under the legal authorisation of the Veterinary Services of the Canton Berne.

\section{Enzyme-linked immunoabsorbent assay of antibodies}

Anti-TETRAVAC antibody levels in blood plasma were determined by sandwich enzyme-linked immunoabsorbent assay (ELISA). For the solid phase, we used a microtitre plate (Corning 96 well flat-bottom, Sigma, Buchs, Switzerland). Each well was coated with a TETRAVAC vaccine dilution ( $200 \mu \mathrm{l}$ diluted in $10 \mathrm{ml}$ of PBS) and incubated for $2 \mathrm{~h}$ at room temperature. The plates were then washed five times with PBS-tween 0.05\%, hereafter denoted PBS-tween (Tween 20, Reactolab,
Servion, Switzerland). We saturated each plate with $200 \mu \mathrm{l}$ of PBStween containing $5 \%$ of milk (PBS-milk, Blotting Grade Blocker not fat dry Milk, Bio-Rad, Switzerland) for $2 \mathrm{~h}$ at room temperature and washed again. Then, $100 \mu \mathrm{l}$ of the diluted plasma (1:100) was randomly distributed into the wells and incubated overnight at $4{ }^{\circ} \mathrm{C}$. After washing, $100 \mu \mathrm{l}$ of peroxydase-conjugated rabbit anti-chicken IgG (1:3000, Sigma, A-9046, Martinez et al., 2003) in PBS-milk was added and left for $2 \mathrm{~h}$ at room temperature. After washing five times, $100 \mu \mathrm{l}$ of peroxydase substrate (o-phenylenediamine dihydrochlorides, $0.4 \mathrm{mg} / \mathrm{ml}$, Sigma) was added for $15 \mathrm{~min}$ at room temperature and then stopped using $50 \mu \mathrm{l}$ of hydrochloric acid ( $\mathrm{HCl} 1 \mathrm{M})$. Optical density (OD; relative measure of anti-TETRAVAC antibody concentration) was read at $490 \mathrm{~nm}$. As a standard, a mixture of positive plasma pools from Alpine swifts was measured in serial dilutions. Anti-TETRAVAC antibody production of each nestling was calculated as its levels of anti-TETRAVAC antibodies at days 20 and 40 minus preexisting anti-TETRAVAC antibodies (i.e. day 15).

\section{Enzyme immunoassay of corticosterone}

Plasma corticosterone concentration was determined using an enzyme immunoassay (EIA; Munro and Stabenfeldt, 1984; Munro and Lasley, 1988). Five-microlitre plasma was added to $195 \mu \mathrm{l}$ water, and from this solution corticosterone was extracted with $4 \mathrm{ml}$ dichloromethane, which was re-dissolved in phosphate buffer and measured in triplicates in the enzyme-immunoassay. The dilution of the corticosterone antibody (Chemicon; cross-reactivity: 11-dehydrocorticosterone $0.35 \%$, Progesterone $0.004 \%, 18-\mathrm{OH}-\mathrm{DOC} 0.01 \%$, Cortisol $0.12 \%, 18-\mathrm{OH}-\mathrm{B} 0.02 \%$ and Aldosterone $0.06 \%$ ) was $1: 8,000$. Horse radish peroxidase (HRP, 1:400,000) linked to corticosterone served as enzyme label and 2,2'Azino-bis(3-ethylbenzo-thiazoline-6-sulfonicacid)diammonium salt (ABTS) as substrate. The concentration of corticosterone in plasma samples was calculated by using a standard curve run in duplicate on each plate. Altogether nine plates were run on three different days. One standard chicken plasma was run in duplicate on each plate to calculate the intra- and inter-assay variation. Intra-assay variation was $7.52 \%$ and inter-assay variation $9.9 \%$. The detection limit of the assay was $1.2 \mathrm{ng} / \mathrm{ml}$.

\section{Weather records}

Alpine swifts can cover long distances (probably up to more than $30 \mathrm{~km}$; Arn, 1960) during a single foraging trip, and thus we gathered weather records from two Swiss meteorological stations (Bern and Neuchâtel) located at about $20 \mathrm{~km}$ from Bienne. For each meteorological station, we obtained information on mean temperature, sum of precipitation and strongest wind gust over periods of $6 \mathrm{~h}$. To investigate how quickly nestling Alpine swifts adjusted their baseline corticosterone levels to weather conditions, we computed mean weather records during the morning (8:00-13:00), the night (20:007:00) and the day preceding (20:00-19:00) the day of blood sampling. For each time period, we calculated the first component (PC1) of a principal components analysis with the three mean meteorological records as loading factors. PC $1_{\text {morning }}$ explained $60.3 \%$ of the total variance in morning weather data (factor loading: minimum temperature: -0.59 ; sum of precipitation: 0.58 ; strongest wind gust: 0.56$), P C 1_{\text {night }}$ explained $45.4 \%$ of the total variance in night weather data (factor loading: minimum temperature: -0.53 ; sum of precipitation: 0.69 ; strongest wind gust: 0.49 ), and $\mathrm{PC} 1_{\mathrm{day}-1}$ explained $50.7 \%$ of the total variance in weather data during the day preceding the day of blood sampling (factor loading: minimum temperature: -0.66 ; sum of precipitation: 0.68 ; strongest wind gust: 0.33). A high PC1 value indicates a cold and rainy period with strong winds, and a small PC1 value indicates a warm and dry period with weak winds. We examined effects of weather conditions on nestling development and corticosterone by including those three PC1 values 
in the starting statistical models. If nestlings show quick adjustment of baseline corticosterone levels to current weather, we predicted that baseline levels would be more strongly associated with weather measured on the morning of blood sampling rather than on the night or on the day preceding the day of blood sampling.

\section{Statistical analyses}

Data were analysed using mixed models with brood size treatment (reduced vs. enlarged), nestling age (day 20 vs. day 40), and weather conditions in the morning, night and day preceding the day of blood sampling entered as five fixed variables in the models. To take into account that more than one nestling per brood were measured and that the same individuals were measured at days 20 and 40, we entered as random factors brood identity nested within brood size treatment as well as nestling identity nested within brood identity. Exploratory analyses provided no evidence that baseline corticosterone levels are differently regulated in male and female Alpine swift nestlings (effect of sex alone or in interaction with treatment or weather indices, all $P$-values $>0.20$ ). Thus, we did not include the effect of sex in our final statistical models. As already reported in other species (e.g. Müller et al., 2009) corticosterone levels did not increase with time if blood was sampled within 3 min after the initial disturbance; $3 \mathrm{~min}$ after initial disturbance there was a steady increase in baseline corticosterone levels with time (handling time: $F_{1,117}=0.17, P=0.68$; handling time ${ }^{2}: F_{1,117}=5.69, P=0.019$; Fig. 1 ). To focus our investigation on baseline corticosterone levels, we discarded from the final analyses 15 corticosterone measurements collected more than $3 \mathrm{~min}$ after initial disturbance (4 out of 40 measurements from nestlings in reduced broods and 11 out of 84 measurements from nestlings in enlarged broods; Fischer exact test: $P=0.77$ ). After the exclusion of these 15 corticosterone measurements, baseline corticosterone levels no longer varied with handling time $\left(F_{1,102}=1.07, P=0.30\right)$ and handling time $^{2}\left(F_{1,102}=1.08\right.$, $P=0.30$ ). The inclusion of these 15 corticosterone measurements in our statistical model, controlling for handling time and handling time ${ }^{2}$ as covariates, provided qualitatively similar results (not shown). Exploratory analyses showed that one 20-day-old nestling reared in an enlarged brood and blood sampled within $32 \mathrm{~s}$ after initial disturbance showed an acute stress response with $108.96 \mathrm{ng} / \mathrm{ml}$ of circulating corticosterone. This elevated corticosterone value may reflect a transitory health problem, which is backed up by the observation that at day 20 this individual was by far the lightest

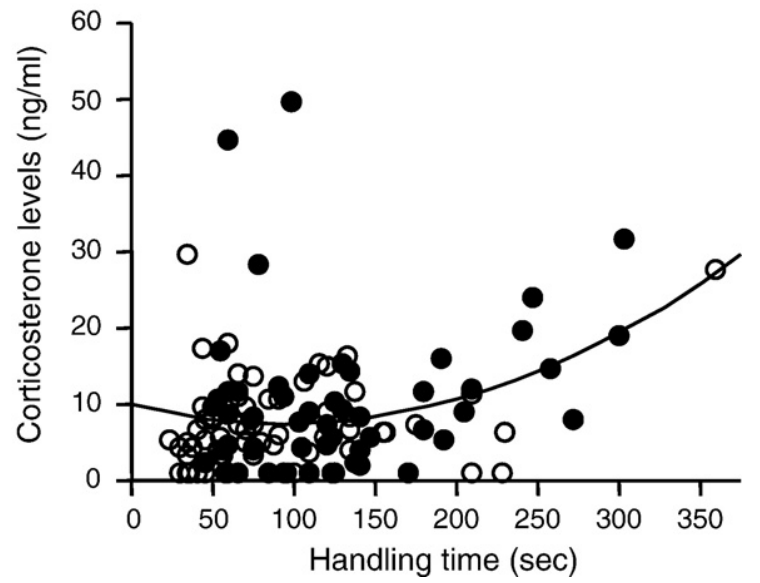

Fig. 1. Nestling corticosterone levels in relationship to handling time after initial disturbance. Nestlings were blood sampled at days 20 and 40 (open and close circles, respectively). The quadratic regression curve between corticosterone levels and handling time is presented. We discarded from the final analyses presented in the Results the 15 corticosterone measurements collected more than 3 min (i.e. 180 s) after initial disturbance. nestling in the present study (59.9 $\mathrm{g}$ against $77.0 \pm 1.1 \mathrm{~g}$ [mean $\pm \mathrm{SE}]$ for the other nestlings reared in enlarged broods). At day 40 , it showed very low corticosterone value $(1.1 \mathrm{ng} / \mathrm{ml})$ and was heavy for its age (107.3 $\mathrm{g}$ against $88.6 \pm 2.5 \mathrm{~g}$ for the other nestlings reared in enlarged broods). This acute (outlier) corticosterone value was removed from the analyses presented below since our aim is to examine baseline levels rather than acute levels of corticosterone. Louse-fly load $(x)$ was box-cox $(x+1)$-transformed before the analyses to ensure the normality of the residuals extracted from our statistical model. Starting models contained the five main effects plus the four possible pairwise interactions between treatment and the four remaining main effects. Nonsignificant fixed variables, starting with nonsignificant interactions, were dropped in a backward fashion from the analyses until the single best model was found; cut-off $P$ value for removal was set to $P=0.05$. Satterthwaite correction was used to approximate the degrees of freedom for fixed effects. We also explored covariations between nestling baseline corticosterone, morphology and immunity using Pearson's correlations. We predicted baseline corticosterone levels to be negatively correlated with nestling body mass, wing length, sternum length and immunity. We have shown elsewhere that in response to poor weather conditions nestlings exhibited a hierarchy of tissues preservation and compensation, with body mass being restored quickly after the end of the period of undernutrition, acceleration of skeletal growth occurring later in development, and compensation in wing length occurring mostly due to a prolongation of growth and delayed fledging (Bize et al. 2006). Hence, we analysed the effects of brood size manipulation and weather conditions on those three traits separately. Statistical analyses were performed using the statistical package JMP IN 7.0 (SAS Institute Inc., Cary, NC, USA).

\section{Results}

Effects of brood size manipulation and weather on nestling development and corticosterone levels

The brood size manipulation experiment significantly affected nestling development, with young reared in enlarged broods being lighter and smaller (in terms of wing length and sternum length) at days 20 and 40 than young reared in reduced broods (significant 'treatment' effect in Table 1; Fig. 2). Nestling body mass and wing length, but not sternum length, were negatively affected by poor weather conditions the day prior to the day of measurements, with nestlings being lighter and having shorter wings when measured after cold and rainy days with strong winds than after warm and dry days with weak winds (significant 'preceding day weather' effect in Table 1). We found no effect of brood size manipulation treatment and weather on nestling louse-fly load and ability to mount a humoral immune response against the vaccine TETRAVAC (Table 1). AntiTETRAVAC antibody production increased significantly with age (Table 1), showing that Alpine swift nestlings were mounting an effective humoral immune response against the vaccine TETRAVAC.

Although the brood size manipulation experiment significantly affected nestling development, corticosterone levels did not differ between nestlings reared in reduced and enlarged broods (Table 1; Fig. 3). To further investigate whether increased baseline corticosterone levels are detected only at the two extremes of our brood size manipulation experiment, we compared baseline corticosterone levels between offspring raised in broods of one vs. four nestlings. Nestlings reared alone, and thus facing no sibling competition, had similar baseline corticosterone levels (mean \pm SE: $6.34 \pm 4.35 \mathrm{ng} / \mathrm{ml}$ ) as nestlings reared in broods of four nestlings $(7.16 \pm 4.98 \mathrm{ng} / \mathrm{ml}$; $F_{1,26.4}=0.00, P=0.99$ ). Effect size calculated from means and standard deviations were moderate (Cohen's $d=0.60$; Cohen, 1969), suggesting that the absence of significant differences in baseline corticosterone levels between treatments was not due to small sample sizes and a lack 
Table 1

Results of mixed models testing the fixed effects of treatment (experimentally reduced vs. enlarged broods), age (day 20 vs. day 40 post-hatching) and weather condition on nestling body mass, wing length, sternum size, louse-fly load, humoral immune response against TETRAVAC and corticosterone levels. Weather condition is divided in morning weather, night weather and preceding day weather condition of the day of blood sampling. Non-significant fixed terms (shown in brackets) were backward dropped from the final models. To control for pseudo-replication, brood identity nested within treatment and nestling identity nested within brood identity were entered in the models as two random factors (statistics not shown).

\begin{tabular}{|c|c|c|c|}
\hline Variables & $d f$ & $F$ & $P$ \\
\hline \multicolumn{4}{|l|}{ Body mass } \\
\hline Treatment & $1,27.3$ & 5.63 & 0.025 \\
\hline Age & $1,60.2$ & 115.74 & $<0.0001$ \\
\hline (Morning weather & $1,62.4$ & 0.01 & $0.92)$ \\
\hline (Night weather & $1,66.3$ & 2.42 & $0.12)$ \\
\hline Preceding day weather & $1,74.5$ & 41.83 & $<0.0001$ \\
\hline \multicolumn{4}{|l|}{ Wing length } \\
\hline Treatment & $1,29.7$ & 4.19 & 0.049 \\
\hline Age & $1,51.2$ & 7281.17 & $<0.0001$ \\
\hline (Morning weather & $1,50.2$ & 0.03 & $0.86)$ \\
\hline (Night weather & $1,52.9$ & 0.11 & $0.74)$ \\
\hline Preceding day weather & $1,57.1$ & 22.34 & $<0.0001$ \\
\hline \multicolumn{4}{|l|}{ Sternum length } \\
\hline Treatment & $1,26.9$ & 6.68 & 0.014 \\
\hline Age & $1,50.5$ & 2473.78 & $<0.0001$ \\
\hline (Morning weather & $1,62.3$ & 0.30 & $0.59)$ \\
\hline (Night weather & $1,71.9$ & 1.63 & $0.21)$ \\
\hline (Preceding day weather & $1,70.7$ & 0.13 & $0.72)$ \\
\hline \multicolumn{4}{|l|}{ Louse-fly load } \\
\hline (Treatment & $1,26.8$ & 1.56 & $0.22)$ \\
\hline (Age & $1,73.64$ & 0.27 & $0.61)$ \\
\hline (Morning weather & $1,73.7$ & 0.67 & $0.42)$ \\
\hline (Night weather & $1,77.3$ & 1.32 & $0.26)$ \\
\hline (Preceding day weather & $1,79.6$ & 0.32 & $0.57)$ \\
\hline \multicolumn{4}{|l|}{ Anti-TETRAVAC antibodies } \\
\hline (Treatment & $1,32.6$ & 0.40 & $0.53)$ \\
\hline Age & $1,61.1$ & 123.18 & $<0.0001$ \\
\hline (Morning weather & $1,30.8$ & 0.04 & 0.84 ) \\
\hline (Night weather & $1,53.6$ & 0.26 & $0.61)$ \\
\hline (Preceding day weather & $1,38.7$ & 1.19 & $0.28)$ \\
\hline \multicolumn{4}{|l|}{ Corticosterone levels } \\
\hline (Treatment & $1,28.5$ & 0.02 & $0.90)$ \\
\hline (Age & $1,77.2$ & 0.19 & $0.66)$ \\
\hline Morning weather & $1,78.7$ & 10.84 & 0.0015 \\
\hline (Night weather & $1,81.2$ & 0.53 & $0.47)$ \\
\hline (Preceding day weather & $1,82.5$ & 0.18 & $0.67)$ \\
\hline
\end{tabular}

of statistical power. Nestling corticosterone levels were significantly affected by weather conditions during the morning of blood sampling (Table 1; Fig. 4) but not by weather conditions during the night or the day preceding the day of blood sampling (Table 1 ). Nestlings had higher corticosterone levels following cold and rainy mornings with strong winds than during warm and dry mornings with weak winds (Fig. 4). There was no significant difference in baseline corticosterone levels between nestlings at 20 vs. 40 days of age (mean \pm SE: $7.90 \pm 1.24$ vs. $8.61 \pm 1.36 \mathrm{ng} / \mathrm{ml}$; Table 1$)$.

\section{Covariation between corticosterone levels and nestling phenotype}

At days 20 and 40, there was no correlation between corticosterone levels and nestling body mass, wing length, sternum length, louse-fly load, and antibody production against the vaccine TETRAVAC (Table 2). Mixed model analyses where brood identity is entered as a random factor to control for the non-independence of nestlings from the same brood provide qualitatively similar results to those shown in Table 2 (results not shown).

\section{Discussion}

In the present study, we aimed to test whether nestling Alpine swifts respond to stressful environmental factors, measured by a
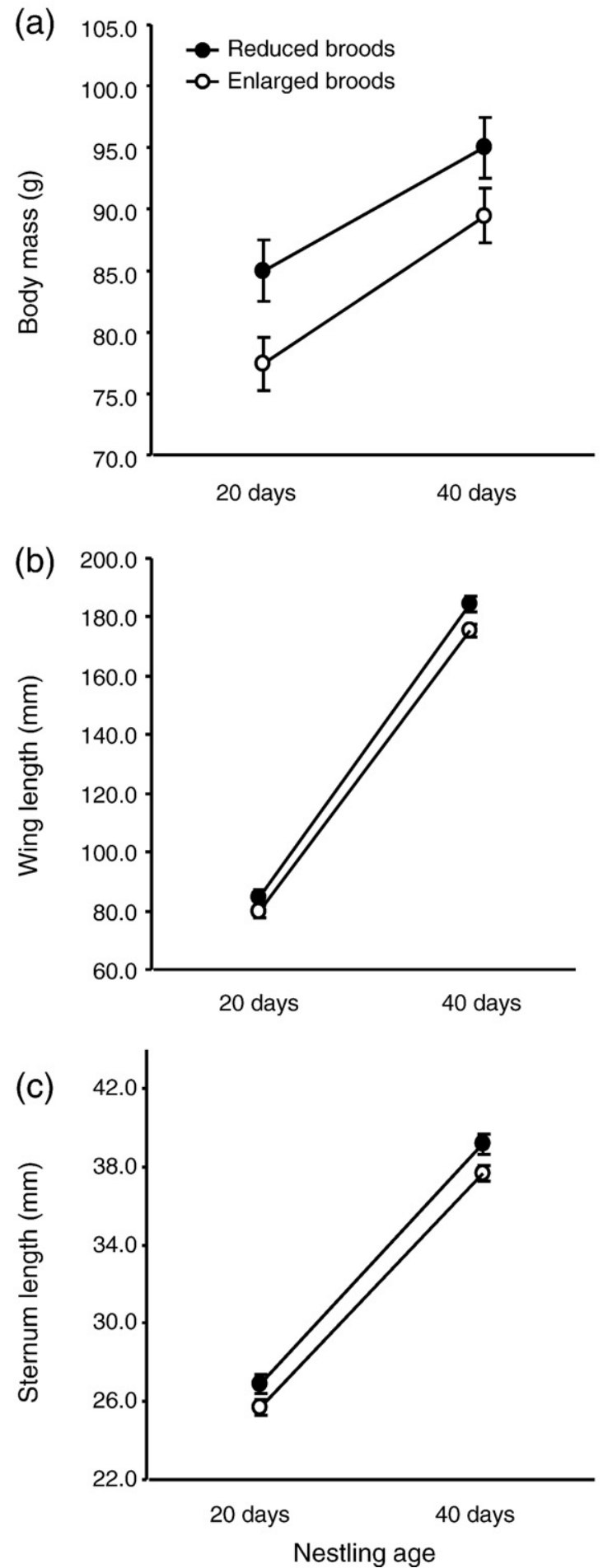

Fig. 2. Least square mean $\pm 1 \mathrm{SE}$ nestling body mass (a), wing length (b) and sternum length (c) in relation to age and brood size manipulation experiment. Least square mean nestling phenotypic values are extracted from mixed models presented in Table 1.

brood size manipulation experiment and by inclement weather conditions, with an elevation of baseline corticosterone levels. Our results provide correlative evidence that nestling baseline corticosterone levels show an elevation in response to unpredictable weather deterioration. We found no experimental evidence that variation in brood size, and by extension sibling competition, is associated with higher baseline corticosterone levels. They suggest that nestling Alpine swifts can quickly modulate their baseline corticosterone 


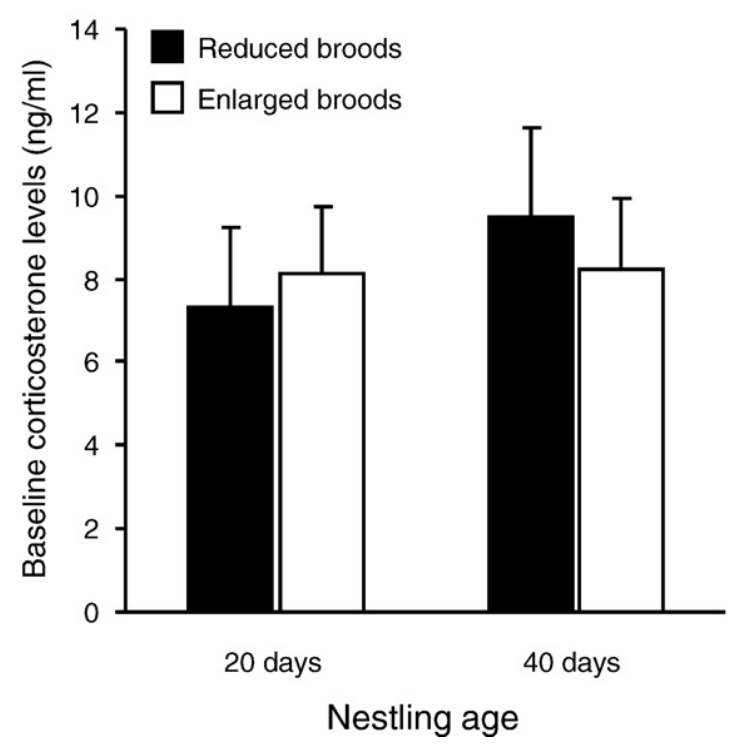

Fig. 3. Least square mean $\pm 1 \mathrm{SE}$ baseline corticosterone levels in 20- and 40-day-old nestlings in relation to the brood size manipulation experiment. Least square mean corticosterone values are extracted from a mixed model where brood size treatment nestling age and morning weather condition were entered as three fixed factors plus the interaction between brood size treatment and age, and nestling identity and brood identity were entered as two random factors.

levels to regulate energy expenditure in response to unpredictable and sudden meteorological perturbations which have clear effects on their food intake and development (Bize et al., 2006; Bize et al., 2007). However, our results provide no evidence that baseline corticosterone is associated with brood size, a life-history trait that also affects nestling food intake and development (Bize and Roulin, 2006a; Roulin et al., 2008; present study). We also did not detect any covariation between baseline corticosterone levels, humoral immune responses and parasite infestation rates.

Brood size manipulation experiments have repeatedly demonstrated that altricial offspring in enlarged broods beg more intensely but monopolise less food than offspring in reduced broods (e.g. Leonard et al., 2000). As a consequence, offspring in experimentally enlarged broods usually have less food to allocate into growth and survival than individuals raised in experimentally reduced broods.

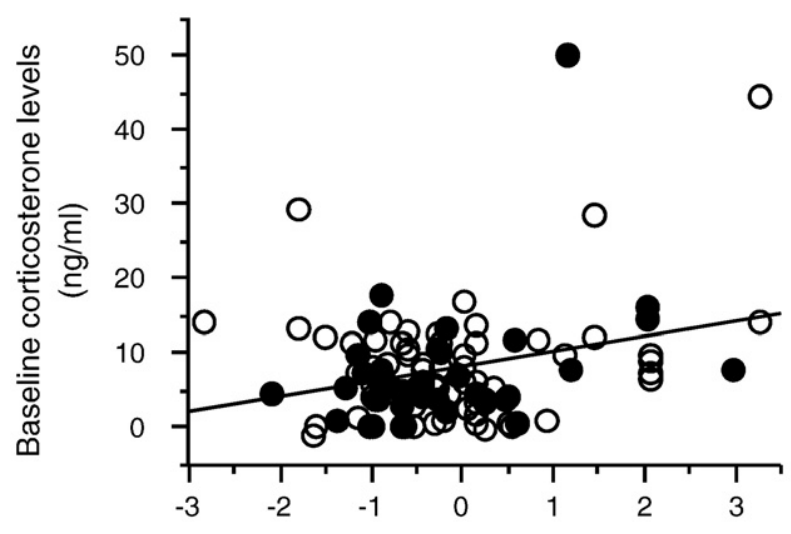
Dry, warm \& weak winds
Weather index (PC1)
Wet, cold \& strong winds

Fig. 4. Nestling baseline corticosterone levels in relationship to morning weathe condition. Nestlings were reared in experimentally reduced or enlarged broods (closed and open circles, respectively). The linear regression line between baseline corticosterone levels and weather condition is presented. Nestling baseline corticosterone levels are leverage residuals extracted from the mixed model presented in Table 1.
Table 2

Pearson's coefficients of correlations between corticosterone levels, nestling morphology, ectoparasite load (i.e. number of louse-flies naturally located in the plumage of nestlings) and humoral immunity (i.e. amount of antibodies produced by nestlings against the vaccine TETRAVAC) at days 20 and 40 after hatching. We did not have enough plasma to measure the production of anti-TERAVAC antibodies for two nestlings at days 20 and 40 after hatching.

\begin{tabular}{|c|c|c|c|c|c|c|}
\hline \multirow[t]{2}{*}{ Variable } & \multicolumn{3}{|c|}{ Day 20} & \multicolumn{3}{|l|}{ Day 40} \\
\hline & $r$ & $n$ & $P$ & $r$ & $n$ & $P$ \\
\hline Body mass & 0.12 & 60 & 0.35 & -0.02 & 45 & 0.90 \\
\hline Wing length & 0.13 & 60 & 0.32 & 0.05 & 45 & 0.73 \\
\hline Sternum length & 0.12 & 60 & 0.36 & -0.12 & 45 & 0.44 \\
\hline Louse fly load & 0.11 & 60 & 0.41 & 0.09 & 45 & 0.57 \\
\hline Anti-TETRAVAC antibodies & 0.04 & 58 & 0.74 & -0.04 & 43 & 0.80 \\
\hline
\end{tabular}

Accordingly, our experiment was successful in inducing two groups of nestlings with distinct growth trajectories between days 20 and 40 after hatching (Fig. 2) and mortality rates (present study; Bize and Roulin, 2006a). Elevated baseline corticosterone levels have been found to down-regulate nestling growth and to trigger higher begging rates (Kitaysky et al., 2001b), two features that may help nestlings to match their growth trajectory and behaviour to food levels and social conditions encountered in enlarged broods. Hence, it has been hypothesised that nestlings should have higher baseline corticosterone levels in enlarged than reduced broods (Saino et al., 2003; Gil et al., 2008). Experimental support for this hypothesis has been given in the barn swallow (Saino et al., 2003), while no difference in baseline corticosterone levels between brood size treatments was detected in nestling spotless starlings (S. unicolor; Gil et al., 2008), nestling blue tits (C. caeruleus; Lobato et al., 2008) and nestling Alpine swifts (present study). Inter-specific variation in baseline corticosterone in response to brood size manipulation may result from various factors, such as the severity of the phenotypic effects induced by brood size manipulation, the sensitivity to food shortage of the species in question (Gil et al., 2008), and inter-specific variation in habituation, desensitisation to the manipulation, or physiological exhaustion because of the manipulation (Cyr and Romero, 2009). The Alpine swift produces smaller broods (mean number of hatchlings $=2.4$ ) than the barn swallow (4.4; Saino et al., 2003), the spotless starling (4.0; Gil et al., 2008) and the blue tit (7.9; Lobato et al., 2008). Thus, increasing or reducing brood sizes by one (or two) nestling(s) leads to a proportionally more severe brood size modification in the Alpine swift than in the species mentioned above (i.e. an increase in brood size of $41.7 \%$ in the Alpine swift, $22.7 \%$ in the barn swallow, $25.0 \%$ in the spotless starling and $12.7 \%$ in the blue tit) which discards the hypothesis that our brood size experiment lacked the strength to induce a shift in body condition which in turn may have altered baseline corticosterone levels. In the same vein, comparison of baseline corticosterone levels between offspring raised alone or in broods of four nestlings showed no difference. Furthermore, nestling Alpine swifts and barn swallows are fed exclusively with aerial insects caught by parents on the wing. Hence, nestlings of both species often have to fast during prolonged periods, and thereby they are expected to be similarly sensitive to periods of food shortage and to have evolved similar response mechanisms to overcome periods of food shortage (Bize et al., 2006, 2007). Note also that, although poor weather conditions and concomitant food depletion can exacerbate sibling competition, we found no evidence that baseline corticosterone levels were modulated by our brood size manipulation experiment in concert with weather conditions. Finally, we found no difference over time in baseline corticosterone levels between brood size treatments, providing no evidence of habituation, desensitisation to the manipulation, or physiological exhaustion because of the manipulation (Cyr and Romero, 2009). Yet, it has been previously demonstrated that brood size manipulation had no significant effect on the probability of offspring to switch nest (Bize 
and Roulin, 2006b). Thus, we cannot exclude that nestling Alpine swifts show little behavioural and hormonal responses to high levels of sibling competition.

In agreement with previous studies showing elevated baseline corticosterone levels in response to unpredictable food shortages in nestling and adult birds (Jenni-Eiermann et al., 2008; Kitaysky et al., 1999b; Kitaysky et al., 2001a; Lobato et al., 2008; Pravosudov et al., 2001; Pravosudov and Kitaysky, 2006; but see Kitaysky et al., 2005; Romero et al., 2000), we observed that nestling Alpine swifts had higher baseline corticosterone levels following cold, rainy and windy mornings which prevented parents from foraging for food. Note that, in addition to food shortage, exposure to cold ambient temperatures during inclement weather might further exacerbate the effect of food shortage on corticosterone secretion by the HPA axis, as previously demonstrated in laboratory rats (Tang et al., 1984). Interestingly, variation in nestling baseline corticosterone levels was related only to the weather in the morning of blood sampling and not to weather in the night or in the day preceding the day of sampling. Thus, in contrast to the idea that baseline corticosterone levels fluctuate in response to prolonged periods of inclement weather (i.e. several days to weeks; Lobato et al. 2008) or even to longer changes in climatic conditions (Romero, 2002), our results indicate fast adjustment of baseline corticosterone levels to changes in weather conditions (see also JenniEiermann et al., 2008). Subtle rapid elevation of baseline corticosterone levels in response to weather deterioration may have at least adaptive functions in nestling Alpine swifts. Firstly, it may promote the catabolism of body reserves to overcome food shortages (Landys et al., 2006; Sapolsky et al., 2000), secondly it may increase begging rates to stimulate food provisioning by parents (Kitaysky et al., 2001b; Quillfeldt et al., 2006), and finally it may regulate hypothermia as an energy conservation mechanism (Hiebert et al., 2000; Bize et al., 2007).

Baseline corticosterone levels are often negatively correlated with body condition (body mass or body reserves), with low body condition caused by fasting being associated with greater needs to catabolise body reserves to fuel the metabolism (e.g. Jenni-Eiermann et al., 2008; Kitaysky et al., 1999a; but see e.g. Criscuolo et al., 2006; Lynn et al., 2003; Pravosudov et al., 2001 for exceptions). Here, we found no significant relationship between nestling body mass and baseline corticosterone levels at days 20 and 40 after hatching, thus providing no evidence for a direct link between body condition and corticosterone secretion despite large variance in nestling body mass (range of body mass in 20-day-old nestlings: 64.0 to 99.0 g; 40-dayold nestlings: 59.4 to $112 \mathrm{~g}$ ). Furthermore, although increased stressinduced and/or baseline circulating levels of corticosterone can be immunosuppressive (Bourgeon and Raclot, 2006; Sapolsky et al., 2000), we found no significant relationship between baseline corticosterone levels and antibody production against a vaccine or between baseline corticosterone levels and natural levels of infestation by a blood sucking ectoparasite. It suggests that elevated baseline corticosterone levels in response to weather deterioration did not come at a cost of humoral immunity. Note however that experiments where offspring were given corticosterone implants provide mixed evidence for an immunosuppressive effect of elevated levels of exogenous baseline corticosterone. An elevation of corticosterone levels depressed the humoral immune response but not the innate immune response in barn owl nestlings (Tyto alba; Stier et al., 2009) and the cutaneous immune response but not the humoral immune response in American kestrel nestlings (Falco sparverius; Butler et al., 2010). Thus, measurements on other branches of the immune system would be necessary to firmly demonstrate that the elevation of baseline corticosterone levels in response to weather deterioration of Alpine swift nestlings did not come at a cost of immunity. It is also not excluded that longer periods of more severe weather deterioration may lead to greater up-regulation of baseline corticosterone levels and, in turn, down-regulation of nestling immunity and greater catabolism of energy reserves. Finally, although circulating corticosterone levels and the ability to respond to a stressor were found to increase with age in developing birds ('the developmental hypothesis'; Sims and Holberton, 2000), we found no evidence for an increase in baseline corticosterone levels on Alpine swift nestlings between 20 and 40 days of age. However, we did not assess the ability of nestlings to respond to a stressor. Because one hypothesis is that increase in baseline corticosterone levels could trigger fledging (Corbel and Groscolas, 2008), and fledging does not occur before 50 days of age in the Alpine swift (Bize et al., 2006), it would have been interesting to assess Alpine swift nestlings HPA responses over a longer developmental period.

In conclusion, our study shows that a rapid but moderate elevation of baseline corticosterone levels may help nestlings to cope with sudden weather deterioration while avoiding paying the cost associated with high levels of circulating corticosterone at least in terms of immunity. Repeated sampling of baseline corticosterone in the same individuals over the course of weather deterioration may provide interesting insights into the links between baseline corticosterone levels, body reserves and immunity. Measures of nestling begging rates may also shed light on the importance of baseline corticosterone in mediating the allocation of resources to behaviour traits vs. growth and immunity.

\section{Acknowledgments}

We are grateful to Lukas Jenni and two anonymous reviewers for helpful comments on the manuscript, the Swiss Meteorological Institute for providing us meteorological records, and the Swiss National Science Foundation for financial support (grant no. PP00A109009 to PB and no. PP00A-102913 to AR).

\section{References}

Arn, H., 1960. Biologische Studien am Alpensegler. Verlag Vogt-Schild AG, Solothurn.

Bize, P., Klopfenstein, A., Jeanneret, C., Roulin, A., 2007. Intra-individual variation in body temperature and pectoral muscle size in nestling Alpine swifts Apus melba in response to an episode of inclement weather. J. Ornithol. 148, 387-393.

Bize, P., Metcalfe, N.B., Roulin, A., 2006. Catch-up growth strategies differ between body structures: interactions between age and structure-specific growth in wild nestling Alpine Swifts. Funct. Ecol. 20, 857-864.

Bize, P., Roulin, A., 2006a. Sibling competition and the risk of falling out of the nest. Anim. Behav. 72, 539-544.

Bize, P., Roulin, A., 2006b. No experimental evidence that sibling competition induces young to switch nest in the colonial Alpine swift Apus melba. Anim. Behav. 72, 869-873.

Bize, P., Roulin, A., Bersier, L.-F., Pfluger, D., Richner, H., 2003. Parasitism and developmental plasticity in Alpine swift nestlings. J. Anim. Ecol. 72, 633-639.

Bize, P., Roulin, A., Tella, J.L., Bersier, L.-F., Richner, H., 2004. Additive effects of ectoparasites over the reproductive attempts in the long-lived Alpine swifts. J. Anim. Ecol. 73, 1080-1088.

Bize, P., Roulin, A., Tella, J.L., Richner, H., 2005. Female-biased mortality in experimentally parasitized Alpine swift Apus melba nestlings. Funct. Ecol. 19, 405-413.

Blas, J., Baos, R., Bortolotti, Marchant T.A., Hiraldo, F., 2005. A multi-tier approach to identifying environmental stress in altricial nestling birds. Funct. Ecol. 19, 315-322.

Blas, J., Bortolotti, G.R., Tella, J.L., Baos, R., Marchant, T.A., 2007. Stress response during development predicts fitness in a wild, long-lived vertebrate. Proc. Natl. Acad. Sci. USA 104, 8880-8884.

Bonier, F., Martin, P.R., Moore, I.T., Wingfield, J.C., 2009. Do baseline glucocorticoids predict fitness? Trends Ecol. Evol. 24, 634-642.

Bourgeon, S., Raclot, T., 2006. Corticosterone selectively decreases humoral immunity in female eiders during incubation. J. Exp. Biol. 209, 4957-4965.

Breuner, C.W., Patterson, S.H., Hahn, T.P., 2008. In search of relationships between the acute adrenocortical response and fitness. Gen. Comp. Endocrinol. 157, 288-295.

Butler, M.W., Leppert, L.L., Dufty, A.M., 2010. Effects of small increases in corticosterone levels on morphology, immune function, and feather development. Physiol. Biochem. Zool. 83, 78-86.

Cohen, J., 1969. Statistical Power Analysis for the Behavioral Sciences. Academic Press, New York.

Corbel, H., Groscolas, R., 2008. A role for corticosterone and food restriction in the fledging of nestling white storks. Horm. Behav. 53, 557-566.

Criscuolo, F., Bertile, F., Durant, J.M., Raclot, T., Gabrielsen, G.W., Massemin, S., Chastel, O., 2006. Body mass and clutch size may modulate prolactin and corticosterone levels in eiders. Physiol. Biochem. Zool. 79, 514-521. 
Cucco, M., Malacarne, G., Orecchia, G., Boano, G., 1992. Influence of weather conditions on pallid swift Apus pallidus breeding success. Ecography 15, 184-189.

Cyr, N.E., Romero, L.M., 2009. Identifying hormonal habituation in field studies of stress. Gen. Comp. Endocrinol. 161, 295-303.

Gasparini, J., Piault, R., Bize, P., Roulin, A., 2009. Pre-hatching maternal effects inhibit nestling humoral immune response in the tawny owl Strix aluco. J. Avian Biol. 40, 271-278.

Gil, D., Bulmer, E., Celis, P., Puerta, M., 2008. Increased sibling competition does not increase testosterone or corticosterone levels in nestlings of the spotless starling (Sturnus unicolor). Horm. Behav. 54, 238-243.

Grüebler, M.U., Morand, M., Naef-Daenzer, B., 2008. A predictive model of the density of airborne insects in agricultural environments. Agric. Ecosyst. Environ. 123, 75-80.

Hiebert, S.M., Salvante, K.G., Ramenofsky, M., Wingfield, J.C., 2000. Corticosterone and nocturnal torpor in the rufous hummingbird (Selasphorus rufus). Gen. Comp. Endocrinol. 120, 220-234.

Jenni-Eiermann, S., Glaus, E., Grüebler, M., Schwabl, H., Jenni, L., 2008. Glucocorticoid response to food availability in breeding barn swallows (Hirundo rustica). Gen. Comp. Endocrinol. 155, 558-565.

Kitaysky, A.S., Kitaiskaia, E.V., Wingfield, J.C., Piatt, J.F., 2001a. Dietary restriction causes chronic elevation of corticosterone and enhances stress response in red-legged kittiwake chicks. J. Comp. Physiol. B 171, 701-709.

Kitaysky, A.S., Piatt, J.F., Wingfield, J.C., Romano, M., 1999a. The adrenocortical stressresponse of black-legged kittiwake chicks in relation to dietary restrictions. J. Comp. Physiol. B 169, 303-310.

Kitaysky, A.S., Romano, M.D., Piatt, J.F., Wingfield, J.C., Kikuchi, M., 2005. The adrenocortical response of tufted puffin chicks to nutritional deficits. Horm. Behav. 47, 606-619.

Kitaysky, A.S., Wingfield, J.C., Piatt, J.F., 1999b. Dynamics of food availability, body condition and physiological stress response in breeding black-legged kittiwakes. Funct. Ecol. 13, 577-584.

Kitaysky, A.S., Wingfield, J.C., Piatt, J.F., 2001b. Corticosterone facilitates begging and affects resource allocation in the black-legged kittiwake. Behav. Ecol. 12, 619-625.

Landys, M.M., Ramenofsky, M., Wingfield, J.C., 2006. Actions of glucocorticoids at seasonal baseline as compared to stress-related levels in the regulation of periodic life processes. Gen. Comp. Endocrinol. 148, 132-149.

Leonard, M.L., Horn, A.G., Gozna, A., Ramen, S., 2000. Brood size and begging intensity in nestling birds. Behav. Ecol. 11, 196-201.

Lobato, E., Merino, S., Moreno, J., Morales, J., Tomas, G., la Puente, J.M.D., Osorno, J.L. Kuchar, A., Mostl, E., 2008. Corticosterone metabolites in blue tit and pied flycatcher droppings: effects of brood size, ectoparasites and temperature. Horm. Behav. 53 , 295-305.

Love, O.P., Bird, D.M., Shutt, L.J., 2003. Corticosterone levels during post-natal development in captive American kestrels (Falco spaervius). Gen. Comp. Endocrinol. 130, 135-141.

Lynn, S.E., Hunt, K.E., Wingfield, J.C., 2003. Ecological factors affecting the adrenocortical response to stress in chestnut-collared and McCown's longspurs (Calcarius ornatus, Calcarius mccownii). Physiol. Biochem. Zool. 76, 566-576.

Malacarne, G., Cucco, M., Bertolo, E., 1994. Sibling competition in asynchronously hatched broods of the pallid swift (Apus pallidus). Ethol. Ecol. Evol. 6, 293-300.

Martinez, J., Tomás, G., Merino, S., Arriero, E., Moreno, J., 2003. Detection of serum immunoglobulins in wild birds by direct ELISA: a methodological study to validate the technique in different species using antichicken antibodies. Functional Ecology 17, 700-706.

McEwen, B.S., Wingfield, J.C., 2003. The concept of allostasis in biology and biomedicine. Horm. Behav. 43, 2-15.
Müller, C., Almasi, B., Roulin, A., Breuner, C.W., Jenni-Eiermann, S., Jenni, L., 2009. Effects of corticosterone pellets on baseline and stress-induced corticosterone and corticosteroid-binding-globulin. Gen. Comp. Endocrinol. 160, 59-66.

Munro, C.J., Stabenfeldt, G.H., 1984. Development of a microtitre plate enzyme immunoassay for the determination of progesterone. J. Endocrinol. 101, 41-49.

Munro, C.J., Lasley, B.L., 1988. Non-radiometric methods for immunoassay of steroid hormones. In: Albertson, B.D., Haseltine, F.P. (Eds.), Non-radiometric Assays: Technology and Application in Polypeptide and Steroid Hormone Detection. Alan R. Liss, Inc, cop, New York, pp. 289-329.

Pravosudov, V.V Kitaysky, A.S, 2006. Effects of nutritional restrictions during posthatching development on adrenocortical function in western scrub-jays (Aphelocoma californica). Gen. Comp. Endocrinol. 145, 25-31.

Pravosudov, V.V., Kitaysky, A.S., Wingfield, J.C., Clayton, N.S., 2001. Long-term unpredictable foraging conditions and physiological stress response in mountain chickadees (Poecile gambeli). Gen. Comp. Endocrinol. 123, 324-331.

Quillfeldt, P., Masello, J.F., Strange, I.J., Buchanan, K.L., 2006. Begging and provisioning of thin-billed prions, Pachyptila belcheri, are related to testosterone and corticosterone. Anim. Behav. 71, 1359-1369.

Ricklefs, R.E., Wikelski, M., 2002. The physiology/life-history nexus. Trends Ecol. Evol. $17,462-468$.

Romero, L.M., 2002. Seasonal changes in plasma glucocorticoid concentrations in freeliving vertebrates. Gen. Comp. Endocrinol. 128, 1-24.

Romero, L.M., 2004. Physiological stress in ecology: lessons from biomedical research. Trends Ecol. Evol. 19, 249-255.

Romero, L.M., Reed, J.M., Wingfield, J.C., 2000. Effects of weather on corticosterone responses in wild free-living passerine birds. Gen. Comp. Endocrinol. 118, 113-122.

Romero, L.M., Wikelski, M., 2001. Corticosterone levels predict survival probabilities of Galápagos marine iguanas during El Niño events. Proc. Natl. Acad. Sci. USA 98, 7366-7370.

Roulin, A., Gasparini, J., Bize, P., Ritschard, M., Richner, H., 2008. Melanin-based colorations signal strategies to cope with poor and rich environments. Behav. Ecol. Sociobiol. 62, 507-519.

Saino, N., Suffritti, C., Martinelli, R., Rubolini, D., Møller, A.P., 2003. Immune response covaries with corticosterone plasma levels under experimentally stressful conditions in nestling barn swallows (Hirundo rustica). Behav. Ecol. 14, 318-325.

Sapolsky, R.M., Romero, L.M., Munck, A.U., 2000. How do glucocorticoids influence stress responses? Integrating permissive, suppressive, stimulatory, and preparative actions. Endocr. Rev. 21, 55-89.

Sims, C.G., Holberton, R.L., 2000. Development of the corticosterone stress response in young Northern Mockingbirds (Mimus polyglottos). Gen. Comp. Endocrinol. 119, 193-201.

Stier, K.S., Almasi, B., Gasparini, J., Piault, R., Roulin, A., Jenni, L., 2009. Effects of corticosterone on innate and humoral immune functions and oxidative stress in barn owl nestlings. J. Exp. Biol. 212, 2084-2090.

Tang, F., Hsieh, A.C., Lee, C.P., Baconshone, J., 1984. Interaction of cold and starvation in the regulation of plasma corticosterone levels in the male rat. Horm. Metab. Res. 16 , 445-448.

Wada, H., 2008. Glucocorticoids: mediators of vertebrate ontogenetic transitions. Gen. Comp. Endocrinol. 156, 441-453.

Wingfield, J.C., Kitaysky, A.S., 2002. Endocrine responses to unpredictable environmental events: stress or anti-stress hormones? Integ. Comp. Biol. 42, 600-609.

Yang, E.V., Glaser, R., 2002. Stress-induced immunomodulation and the implication for health. Int. Immunopharmacol. 2, 315-324.

Zera, A.J., Harshman, L.G., 2001. The physiology of life history trade-offs in animals. An. Rev. Ecol. Syst. 32, 95-126. 